\title{
Análise de custo e fatores relacionados à devolução de medicamentos à farmácia hospitalar de um hospital privado no Município de Belém
}

\author{
Analysis of cost and factors related to the return of medication to the hospital pharmacy of a \\ private hospital in the City of Belem \\ Análisis de costos y factores relacionados con la devolución de medicamentos a la farmacia \\ hospitalaria de un hospital privado de la Ciudad de Belém
}

Recebido: 11/10/2021 | Revisado: 18/10/2021 | Aceito: 20/10/2021 | Publicado: 23/10/2021

\author{
Maria Pantoja Moreira de Sena \\ ORCID: https://orcid.org/0000-0001-6641-4618 \\ Universidade Federal do Pará, Brasil \\ E-mail: mariapantojamoreira@ hotmail.com \\ Camila Martins Oliveira \\ ORCID: https://orcid.org/0000-0002-6425-2384 \\ Universidade Federal do Pará, Brasil \\ E-mail: cmofarma@gmail.com \\ Clarisse Andrade Sales \\ ORCID: https://orcid.org/0000-0002-9451-666X \\ Universidade Federal do Pará, Brasil \\ E-mail: clarisseasales@gmail.com \\ Marcos Felipe Rodrigues de Souza \\ ORCID: https://orcid.org/0000-0002-6246-7525 \\ Universidade Federal do Pará, Brasil \\ E-mail: marcosfrodrigues.br@gmail.com \\ Renato Bruno Cavalcante de Melo \\ ORCID: https://orcid.org/0000-0002-6159-3802 \\ Universidade Federal do Pará, Brasil \\ E-mail: renatobcdemelo@gmail.com \\ Crystyanne de Sousa Freitas \\ ORCID: https://orcid.org/0000-0003-4279-1587 \\ Universidade Federal do Pará, Brasil \\ E-mail: crystyannefreitas@icloud.com \\ Amanda Gabryelle Nunes Cardoso Mello \\ ORCID: https://orcid.org/0000-0001-7661-1615 \\ Universidade Federal do Pará, Brasil \\ E-mail: agncmello@gmail.com \\ Luann Wendel Pereira de Sena \\ ORCID: https://orcid.org/0000-0001-9363-5766 \\ Universidade Federal do Pará, Brasil \\ E-mail: luannsena@gmail.com
}

\begin{abstract}
Resumo
Objetivo: analisar os motivos e custos relacionados a devolução de medicamentos, após distribuição, para a farmácia do hospital. Métodos: Trata-se de um estudo retrospectivo quantitativo no qual foram avaliados os custos das devoluções procedentes dos pacientes internados nas unidades clínicas e cirúrgicas, através do sistema informatizado de gestão hospitalar SOUL MV ${ }^{\circledR}$. Resultados: estão expressos os motivos e custo compreendidos no regresso desses produtos nos anos 2018, 2019 e 2020, respectivamente. Um total de R \$ 2.870.733,19 foi declarado. Observa-se que em 2018 quando utilizava-se o SDM coletivo, o custo de devolução foi de R\$1.161.956,41, equivalendo a 40,4\% do custo total. Em 2019 e 2020, já com o sistema dose única individualizado, os gastos foram reduzidos para 31,9\% e $27,6 \%$, respectivamente. O aprazamento dos medicamentos foi o mais predominante $(57,1 \%)$, seguida do material em desacordo $(9,2 \%)$, suspenção da prescrição $(8,2 \%)$, material não utilizado $(7,2 \%)$, duplicidade $(6,1 \%)$, recusa do paciente e/ou procedimento $(2,4 \%)$, solicitação incorreta $(2,1 \%)$, óbito $(1,2 \%)$, transferência do paciente $(0,5 \%)$ e procedimento suspenso $(0,2 \%)$. Conclusão: Os resultados do estudo demostram que a utilização do SDM dose unitária individualizado reduziu significativamente os custos de devolução e os erros relacionados aos medicamentos. Essa prática pode trazer economia aos serviços de saúde quando os medicamentos devolvidos estão em condições apropriadas ao reuso por outro paciente. Porém, a reutilização exige discussão e planejamento, pois a segurança e o sucesso dos tratamentos dependem de cuidados durante o armazenamento.
\end{abstract}

Palavras-chave: Medicamentos; Análise de custo; Devolução. 


\begin{abstract}
Objective: analysis of reasons and costs related to the return of medication, after distribution, to the hospital pharmacy. Methods: This is a quantitative retrospective study in which the costs of returns from patients admitted to clinical and surgical units were evaluated, using the computerized hospital management system SOUL MV ${ }^{\circledR}$. Results: the reasons and costs involved in the return of these products in the years 2018, 2019 and 2020, respectively, are expressed. A total of $\mathrm{R} \$ 2,870,733.19$ was declared. It is observed that in 2018 when the collective SDM was used, the return cost was BRL 1,161,956.41, equivalent to $40.4 \%$ of the total cost. In 2019 and 2020, with the single dose individualized system, expenses were reduced to $31.9 \%$ and $27.6 \%$, respectively. The drug deadline was the most predominant $(57.1 \%)$, followed by material in disagreement $(9.2 \%)$, prescription suspension $(8.2 \%)$, unused material (7.2\%), duplicity (6.1\%), patient and/or procedure refusal $(2.4 \%)$, incorrect request $(2.1 \%)$, death $(1.2 \%)$, patient transfer $(0.5 \%)$ and procedure suspended $(0.2 \%)$. Conclusion: The results of the study demonstrate that the use of individualized unit dose SDM significantly reduced return costs and drug-related errors. This practice can bring savings to health services when the returned medications are in a condition suitable for reuse by another patient. However, reuse requires discussion and planning, as the safety and success of treatments depend on care during storage.
\end{abstract}

Keywords: Medicines; Cost analysis; Devolution.

\title{
Resumen
}

Objetivo: análisis de los motivos y costes relacionados con la devolución de la medicación, tras su distribución, a la farmacia hospitalaria. Métodos: Se trata de un estudio cuantitativo retrospectivo en el que se evaluaron los costos de los retornos de los pacientes ingresados en unidades clínicas y quirúrgicas, utilizando el sistema informático de gestión hospitalaria SOUL $\mathrm{MV}^{\circledR}$. Resultados: se expresan las razones y costos que conllevó la devolución de estos productos en los años 2018, 2019 y 2020, respectivamente. Se declaró un total de R $\$ 2.870 .733$, 19. Se observa que en 2018 cuando se utilizó el SDM colectivo, el costo de retorno fue de R\$1.161.956,41, equivalente al 40,4\% del costo total. En 2019 y 2020, con el sistema individualizado de dosis única, los gastos se redujeron al 31,9\% y 27,6\%, respectivamente. El plazo de medicamentos fue el más predominante $(57,1 \%)$, seguido del material en desacuerdo $(9,2 \%)$, suspensión de prescripción $(8,2 \%)$, material no utilizado $(7,2 \%)$, duplicidad $(6,1 \%)$, rechazo del paciente y / o procedimiento. $(2,4 \%)$, solicitud incorrecta $(2,1 \%)$, fallecimiento $(1,2 \%)$, traslado de pacientes $(0,5 \%) \mathrm{y}$ procedimiento suspendido $(0,2 \%)$. Conclusión: Los resultados del estudio demuestran que el uso de dosis unitarias individualizadas SDM redujo significativamente los costos de devolución y los errores relacionados con los medicamentos. Esta práctica puede generar ahorros en los servicios de salud cuando los medicamentos devueltos se encuentran en condiciones adecuadas para que otro paciente los reutilice. Sin embargo, la reutilización requiere discusión y planificación, ya que la seguridad y el éxito de los tratamientos dependen del cuidado durante el almacenamiento.

Palabras clave: Medicamentos; Análisis de costos; Devolución.

\section{Introdução}

Em 2002, o Ministério da Saúde do Brasil (MS) estabeleceu o hospital como uma instituição de saúde desigual de unidades de inúmeros portes, que podem desenvolver uma série de serviços e atividades. Estes executam funções diversas no âmbito da rede de atendimento à saúde e se constituem por oferecer cuidado à saúde de pacientes internados em leitos hospitalares de forma integral. Possuem objetivos mais complexos do que simplesmente curar, neles persistem inúmeras questões de ordem gerencial e assistencial que ecoam na qualidade da atenção à saúde ofertada (Menezes et al., 2021).

O farmacêutico exerce um papel fundamental no desempenho das atividades hospitalares e o paciente é o real beneficiário de suas ações. A assistência farmacêutica (AF) deve ser um conjunto de atitudes, comportamentos, compromissos e responsabilidades. O conceito de AF foi apresentado por Hepler, como um processo cooperativo para provisão responsável da farmacoterapia, com o propósito de alcançar resultados que aprimorem a qualidade de vida do paciente (Conselho Federal de Farmácia, 2017).

A farmácia hospitalar (FH) é uma seção do hospital que têm como finalidade preservar o uso seguro e racional dos medicamentos prescritos e corresponder a demanda de medicamentos dos pacientes hospitalizados (Freitas et al., 2021). A AF constituiu-se como um sistema complexo e relevante no domínio da gestão de sistemas e serviços de saúde, não somente por contemplar um dos insumos básicos para cuidados aos pacientes, como também pelos altos custos envolvidos (Barros et al., 2021). 
Os indicadores de gestão financeira aplicadas na administração de farmácias hospitalares são uma forma segura de monitorar o desempenho e gerenciamento dos processos (Pereira et al., 2019). O conhecimento e a avaliação da atuação dos serviços e da instituição representam um papel expressivo ao confrontar custos e benefícios. Estes têm sido empregues para determinar a atuação de inúmeras atividades produtivas (Andreoli et al., 2015). O progresso dos procedimentos de gestão pela qualidade foi seguido de uma crescente necessidade de coletar, tabular e analisar dados a fim de, identificar desvios e racionalizar processos (Gil et al., 2015). O farmacêutico é o encarregado pela coordenação dos procedimentos administrativos, dos recursos e das pessoas, buscam mecanismos gerenciais que desenvolvam a eficácia dos processos criados pela farmácia. Para que seus objetivos sejam obtidos deve-se possuir um sistema efetivo de informações e, um sistema de controle de acompanhamento de custo, para evitar perda de insumos e econômico (Ferranti, 2017).

A diversidade das terapias medicamentosas e as indicações dos resultados das intervenções farmacêuticas na melhoria dos regimes terapêuticos e na redução dos custos assistenciais ampliam a importância de uma AF de qualidade (Santos et al., 2019). A farmácia tem atuação estratégica na composição de uma política de uso racional de medicamentos (URM) almejando aprimorar e garantir a qualidade da farmacoterapia e reduzir os custos para estabelecimento, já que a politerapia além de acumular os custos com cuidado ao paciente, gera elevação da morbi-mortalidade resultante do uso indevido dos medicamentos (Freitas et al., 2021).

O farmacêutico possui um amplo desafio na administração de suprimentos e no controle correto do estoque para o suporte das reais necessidades do hospital (Silva et al., 2017). O controle efetivo e o aproveitamento de instrumentos para gerir as informações, devem ser acompanhadas da criação de políticas de estoque sendo a organização um dos principais recursos de gestão, para que haja equilíbrio no suprimento de materiais necessários para o atendimento da demanda com o mínimo de custo possível (Gil et al., 2015).

Diante dessas circunstâncias, torna-se fundamental a verificação constante desta temática, no sentido de demonstrar casos de sucesso e insucessos que possam instituir novas perspectivas de soluções da gestão em farmácia, tornando-se considerável ao passo que evidenciará possíveis problemas e facilidades no desenvolvimento das atividades dos gestores.

Neste sentindo, este estudo teve como objetivo realizar a análise dos motivos e custos relacionados a devolução de medicamentos, após distribuição, para a farmácia do hospital.

\section{Metodologia}

Trata-se de um estudo retrospectivo quantitativo no qual foram avaliados os custos das devoluções procedentes dos pacientes internados nas unidades clínicas e cirúrgicas, através do sistema informatizado de gestão hospitalar SOUL MV ${ }^{\circledR}$ (Freitas et al., 2021).

O local de estudo foi um hospital de grande porte que possui mais de seis décadas de existência e atua na região metropolitana de Belém. É um hospital filantrópico, geral, de atenção terciária, multibloco, possuindo 246 leitos, sendo 243 operacionais: clínica médica (116), pediátrica (24), cirúrgica (90) e unidade de terapia intensiva (clínica = 6 + cirurgia = 7), possui percentual médio de ocupação de $68,9 \%=6.816$ entradas de paciente/ano.

Os Dados foram recebidos no serviço de farmácia, o qual funciona 24 horas/dia, atendendo às prescrições dos pacientes internados na instituição, através do sistema coletivo (até/em 2018) e individual e unitário (a partir de 2018/até o momento). O serviço possui 8 (oito) farmacêuticos que exercem rotineiramente as análises das prescrições, antes da dispensação. 


\section{Resultados}

O hospital recebe constantemente pacientes com múltiplos diagnósticos e evidencia-se o crescimento no consumo de medicamentos. Em 2018, o sistema de distribuição de medicamentos (SDM) empregue era o coletivo e posteriormente foi alterado para o sistema dose unitária individualizado. Os medicamentos são acolhidos na Central de Abastecimento Farmacêutico (CAF) e/ou farmácia central do hospital, armazenados e distribuídos para as farmácias satélites com o propósito de compor os volumes presentes no local. Os medicamentos são dispensados para as enfermarias a partir deste estoque.

A devolução de produtos farmacêuticos é acolhida diariamente nas farmácias, onde são quantificadas, avaliadas quanto as conjunturas de integridade, temperatura e validade. Os motivos de retorno dos medicamentos são diversos, tais como: alta do paciente, aprazamento da prescrição, duplicidade, material em desacordo, material não utilizado, óbito, procedimento suspenso, recusa do procedimento, solicitação incorreta, suspensão da prescrição, transferência do paciente.

Na Tabela 1, estão expressos os motivos e custo compreendidos no regresso desses produtos nos anos 2018,2019 e 2020, respectivamente. Um total de R \$2.870.733,19 foi declarado. Observa-se que em 2018 quando utilizava-se o SDM coletivo, o custo de devolução foi de $\mathrm{R} \$ 1.161 .956,41$, equivalendo a 40,4\% do custo total. Em 2019 e 2020 , já com o sistema dose única individualizado, os gastos foram reduzidos para 31,9\% e 27,6\%, respectivamente. O aprazamento dos medicamentos foi o mais predominante $(57,1 \%)$, seguida do material em desacordo $(9,2 \%)$, suspenção da prescrição $(8,2 \%)$, material não utilizado $(7,2 \%)$, duplicidade $(6,1 \%)$, recusa do paciente e/ou procedimento $(2,4 \%)$, solicitação incorreta $(2,1 \%)$, óbito $(1,2 \%)$, transferência do paciente $(0,5 \%)$ e procedimento suspenso $(0,2 \%)$ (Tabela 1$)$.

Tabela 1 - Motivos e custo da devolução dos produtos ao estoque da farmácia no período 2016 a 2018.

\begin{tabular}{|c|c|c|c|c|}
\hline Motivos & 2018 & 2019 & 2020 & Total \\
\hline Alta do Paciente & $87.053,17$ & $43.790,21$ & $23.476,37$ & $154.319,75$ \\
\hline Aprazamento & $512.116,55$ & $598.243,28$ & $529.974,32$ & $1.640 .334,15$ \\
\hline Duplicidade & $96.005,87$ & $53.972,93$ & $27.471,35$ & 177.450,15 \\
\hline $\begin{array}{c}\text { Material em } \\
\text { desacordo }\end{array}$ & $105.766,18$ & $122.183,1$ & $37.705,61$ & $265.654,89$ \\
\hline $\begin{array}{c}\text { Material não } \\
\text { utilizado }\end{array}$ & $208.418,94$ & $\mathrm{NC}$ & NC & 208.418,94 \\
\hline Óbito & $18.381,1$ & $11.087,4$ & $5.692,44$ & $35.160,94$ \\
\hline $\begin{array}{c}\text { Procedimento } \\
\text { suspenso }\end{array}$ & $6.182,06$ & NC & NC & $6.182,06$ \\
\hline $\begin{array}{l}\text { Recusa do paciente } \\
\text { e/ou procedimento }\end{array}$ & $14.694,98$ & $17.582,29$ & $37.332,18$ & $69.609,45$ \\
\hline $\begin{array}{c}\text { Solicitação } \\
\text { Incorreta }\end{array}$ & $24.301,93$ & $13.680,83$ & $23.101,13$ & $61.083,89$ \\
\hline $\begin{array}{c}\text { Suspensão da } \\
\text { prescrição }\end{array}$ & $78.078,77$ & $49.641,47$ & $107.750,72$ & 235.470,96 \\
\hline $\begin{array}{c}\text { Transferência do } \\
\text { paciente }\end{array}$ & $10.956,86$ & $5.655,67$ & 435,48 & 17.048,01 \\
\hline Total & 1.161.956,41 & $915.837,18$ & $792.939,60$ & $2.870 .733,19$ \\
\hline
\end{tabular}

Legenda: AP - alta paciente; APZT - aprazamento; CD - cobrança em duplicidade; MD - material em desacordo; MNT - material não utilizado; OBT - óbito; PS - procedimento suspenso; RP - recusa do paciente e/ou procedimento; SI - solicitação incorreta; SP - suspensão da prescrição; TP - transferência do paciente; NC - não consta. Fonte: Autores (2021). 


\section{Discussão}

A FH é um setor clínico-assistencial, técnica e administrativa, onde se demandam as atividades relacionadas à AF, coordenada puramente por farmacêutico, instituindo a estrutura organizacional do hospital e incorporada funcionalmente com as demais unidades administrativas e de assistência ao paciente (Silva et al., 20210). Suas atribuições devem beneficiar a permanente disponibilidade dos produtos, segundo as necessidades dos pacientes, contribuindo para o URM. Entre as atividades, destacam-se a seleção, programação, aquisição, armazenamento e distribuição, controle de qualidade e utilização (prescrição e dispensação) (Valentin et al., 2021).

Os medicamentos simbolizam um custo elevado nas unidades hospitalares e dispõem grande relevância no tratamento da maioria das doenças, o que justifica a realização de ação e/ou medidas que garantam o uso racional destes produtos (Varallo et al., 2019). Uma das medidas de grande efeito, neste contexto, é uma concreta dispensação e/ou distribuição dos medicamentos que visa, a assistência de forma segura e eficiente dos itens necessários para as unidades ou serviços do hospital. A distribuição aos inúmeros setores do hospital deve ser feita de acordo com uma programação previamente acertada com os serviços. Nesta programação, devem ser ponderados fatores técnicos e administrativos, incluindo também, os de ordem logística e aqueles pertinentes à qualidade do serviço de atenção aos pacientes (Viana et al., 2017).

Outros processos que ocorre é a restituição, ou seja, a devolução dos medicamentos distribuídos às unidades pela FH. Essa tarefa pode suceder por diversos motivos: quando o paciente não faz o uso do medicamento, alta médica, mudança de enfermaria ou óbito (Hohl et al., 2017). O regresso do medicamento à farmácia se faz indispensável tendo em vista que, outro paciente poderá fazer o uso dessa medicação não utilizada, diminuindo assim, os custos hospitalares. Porém, na prática, o estorno pode apresentar uma série de irregularidades, prejudicando a obtenção das vantagens do processo (Leite et al., 2019).

No estudo, foi mencionado que o SDM utilizado foi o coletivo e posteriormente o sistema de dose unitária individualizado. A utilização do SDM dose unitária individualizado reduziu significativamente os custos de devolução e os erros relacionados aos medicamentos. Quanto maior for a eficiência e a eficácia do SDM, melhor será o sucesso das terapias instauradas (Maioli et al., 2018). Após a inserção desse sistema (a partir de 2018), houve uma redução gradativa dos custos envolvendo os motivos de devolução dos medicamentos. O elevado custo pode ser justificado devido a periodicidade em que esta ocorre, pois, é provável que erros ocorram devido devolução atrasada de materiais e medicamentos que ficam reunidos no posto da unidade de enfermagem, sujeitos a evasão ou uso inadequado (Menezes et al., 2021).

Com prescrições integrando combinações cada vez mais complexas, tornou-se difícil distinguir previamente, os potenciais interações medicamentosas (PIM), que são aquelas em que há chance da modificação do efeito dos fármacos envolvidos, podendo levar a resultados não desejados, elevando a incidência de efeitos adversos da terapia, sem acréscimo do benefício terapêutico (Gonçalves et al., 2016).

O aprazamento seguro e categórico de medicamentos é uma importante responsabilidade do profissional enfermeiro e do farmacêutico clínico. A partir do aprazamento, os profissionais constituem o plano terapêutico medicamentoso estabelecido aos pacientes. Em sua maioria, o padrão de intervalos de horários está intimamente associado à rotina de cuidados da enfermagem, de médicos e dos farmacêuticos (Noormandi et al., 2021).

Foi detectado que o aprazamento correspondeu a $57,1 \%$ dos cursos relativos à devolução de produtos e/ou medicamentos para a farmácia. Este alto índice pode ser justificado pela efetiva avaliação da equipe de enfermagem e/ou do farmacêutico clínico que estão realizando este serviço com eficiência (Oliveira et al., 2018). Outros fatores como o material em desacordo, suspensão da prescrição, material não utilizado, duplicidade, recusa do paciente e/ou procedimento, solicitação incorreta, óbito, transferência do paciente e procedimento suspenso, foram constatados em outros estudos. Sabe-se que existem várias razões para a troca do tratamento medicamentoso como as reações adversas, inefetividade não quantitativa, segurança, entre outras. Na farmácia hospitalar, quando o paciente introduz uma nova terapia medicamentosa, eles normalmente 
devolvem os medicamentos utilizados anteriormente, o que justifica a suspensão da prescrição, os materiais não utilizados ou procedimentos suspensos (Barros et al., 2021).

A restituição de medicamentos às farmácias de serviços de saúde é uma questão que precisa ser mais discutida pelas autoridades sanitárias brasileiras. As responsabilidades devem ser assumidas pelos farmacêuticos, profissionais com formação adequada para lidar com questões relacionadas ao uso desses produtos (Conselho Federal de Farmácia, 2017).

A devolução de medicamentos deve ser motivada, pois permite que tenham uma direção final adequada. Essa prática pode trazer economia aos serviços de saúde quando os medicamentos devolvidos estão em condições apropriadas ao reuso por outro paciente. Porém, a reutilização exige discussão e planejamento, pois a segurança e o sucesso dos tratamentos dependem de cuidados durante o armazenamento (Ferranti, 2017).

\section{Conclusão}

Os resultados do estudo demostram que a utilização do SDM dose unitária individualizado reduziu significativamente os custos de devolução e os erros relacionados aos medicamentos. Foi detectado que o aprazamento correspondeu a $57,1 \%$ dos cursos relativos à devolução de produtos e/ou medicamentos para a farmácia. Outros fatores como o material em desacordo, suspensão da prescrição, material não utilizado, duplicidade, recusa do paciente e/ou procedimento, solicitação incorreta, óbito, transferência do paciente e procedimento suspenso, foram constatados em outros estudos. A devolução de medicamentos deve ser motivada, pois permite que tenham uma direção final adequada. Essa prática pode trazer economia aos serviços de saúde quando os medicamentos devolvidos estão em condições apropriadas ao reuso por outro paciente. Porém, a reutilização exige discussão e planejamento, pois a segurança e o sucesso dos tratamentos dependem de cuidados durante o armazenamento.

Para um domínio maior, recomenda-se que seja pontuado os produtos e/ou medicamentos envolvidos na devolução na farmácia, a fim de, analisarmos amplamente todos os processos referentes ao ciclo logístico em um hospital.

\section{Referências}

Andreoli G. L. M., \& Dias C. N. (2015). Planejamento e gestão logística de medicamentos em uma central de Abastecimento Farmacêutico hospitalar. Revista de Administração Hospitalar e Inovação em Saúde, 12 (4): 20-28.

Barros, M. E., \& Araújo, I. G. (2021). Avaliação das intervenções farmacêuticas em unidades de terapia intensiva de um hospital de ensino. Revista Brasileira de Farmácia Hospitalar e Serviços de Saúde, 12 (3): 0561.

Conselho Federal de Farmácia (CFF). Farmácia Hospitalar: coletânea de práticas e conceitos. CFF, 2017.

Ferranti E. (2017). Gestão de estoque de medicamentos utilizando classificação ABC em um hospital público. Perspectiva Econômica, 13 (3): $215-229$.

Freitas, G. R., Santos, J. U., França, J. S., Pinto, R. S., \& Seixas, B. V. (2021). Economic impact of pharmacysts' interventions in asthma management: a systemmatic review. Revista Brasileira de Farmácia Hospitalar e Serviços de Saúde, 21 (3):0587.

Gil R. B., Chaves L. D. P., \& Laus A. M. (2015) Gerenciamento de recursos materiais com enfoque na queixa técnica. Revista Eletrônica de Enfermagem, 17 (1): 100-7.

Gonçalves A. A., Yoshida D., Pitassi C., \& Freitas J. A. S. B. (2016). Fatores críticos de sucesso na gestão da cadeia de suprimentos em saúde pública: uma visão dos gestores dos institutos federais da cidade do Rio de Janeiro, Rj, Brasil. Revista de Administração Hospitalar e Inovação em Saúde, 13 (2): $40-52$.

Hohl, C. M., Partovi N., \& Ghement I (2017). Impact of early in-hospital medication review by clinical pharmacists on health services utilization. PlosOne, 12(2): e0170495.

Leite A. L. A. (2019). Gerenciamento da Terapia Medicamentosa: uma análise de indicadores do serviço farmacêutico. Trabalho de Conclusão de Curso, Universidade Federal de Minas Gerais, MG, Brasil.

Maioli N. A., \& Santos H. C. B. (2018). Intervenções Farmacêuticas e sua importância na Segurança do Paciente Hospitalizado. Colloq Vitae, 10 (2): 35-40.

Menezes K. B., Silva A. S., Cunha-Matos C. E., Nutels T., Oliveira A. D., Neves S. J., \& Leopardi-Gonçalves M. G. (2021). Associação entre discrepâncias medicamentosas e o tempo de internação na clínica cirúrgica em um hospital universitário. Revista Brasileira de Farmácia Hospitalar e Serviços de Saúde, 12 (2): 0547 .

Noormandi A., Karimzadeh I., Mirjalili M., \& Khalili H. (2021). Clinical and economic impacts of clinical pharmacists' interventions in Iran: a systematic review. Daru, 27 (1): 361-378. 
Research, Society and Development, v. 10, n. 14, e01101421645, 2021

(CC BY 4.0) | ISSN 2525-3409 | DOI: http://dx.doi.org/10.33448/rsd-v10i14.21645

Oliveira A.M., Rodrigues V.A.V., \& Passerini J. P. (2018). Queixas técnicas e reações adversas a medicamentos notificadas em um hospital regional no Brasil: um estudo transversal. ABCS Health Sciences, 43 (1): 25-29.

Pereira R. M., Felix B. S., Monteiro N. J., \& Fernandes R. M. (2019). Análise da gestão de estoque em uma farmácia hospitalar em Marabá-PA: um estudo de caso. Sistemas \& Gestão, 14 (4): 413-423.

Santos N. S., Marengo L. L., Moraes F. S., \& Barberato. (2019). Intervenções para reduzir a prescrição de medicamentos inapropriados para idosos. Revista de Saúde Pública, 53:7.

Silva P. L., Castilho S. R., \& Ferraz C. V. V. G. (2017). Análise dos Resultados da Aplicação de Práticas Gerenciais na Logística de Estoque de uma Farmácia Hospitalar. Revista de Administração Hospitalar e Inovação em Saúde, 14 (2): 14-31.

Silva R. T. D., Santos F. J. S., Bezerril M. S., Salvador P. (2020). Estratégias de gestão da qualidade e gestão de material de uma farmácia hospitalar: revisão interativa da literatura. Revista de Administração Hospitalar e Inovação em Saúde, 17 (1): 1983-1991.

Valentin A. A. S., Rezende C.P., Nascimento Y. A., Gualberto F. C. M., Mendonça S. A. M., Nascimento M. M. G., \& Oliveira D. R. (2021) Aspectos envolvidos na sustentabilidade do serviço de gerenciamento da terapia medicamentosa. Research Society and Development, 10 (8): e13310817135.

Varallo F. R., Forgerini M., \& Hedeiro M. T. (2019). Harmonization of Pharmacovigilance Regulation in Brazil: Opportunities to improve Risk Communication. Clinical Therapyeutics, 41 (3): 598-602.

Viana S., Arantes T., \& Ribeiro S. (2017). Intervenção do farmacêutico clínico em uma Unidade de Cuidados Intermediários com foco no paciente idoso. Einstein, 15 (3): 283-288. 